\title{
A DIFFUSION EQUATION WITH LOCALIZED CHEMICAL REACTIONS*
}

\author{
by JOHN M. CHADAM and HONG-MING YIN
}

(Received 15th July 1992)

\begin{abstract}
In some chemical reaction-diffusion processes, the reaction takes place only at some local sites, due to the presence of a catalyst. In this paper we study the well-posedneș of a model problem of this type. Sufficient conditions are found to ensure global existence and finite time blowup. The blowup rate and the blowup set are also investigated in the case of special nonlinearity.
\end{abstract}

1991 Mathematics subject classification: 35K 57, 35B05

\section{Introduction}

Let $\Omega$ be a bounded domain in $R^{N}$ with smooth boundary $S=\partial \Omega$. Let $\Gamma$ be a $C^{2}$-hypersurface in $\Omega$. Consider the following problem:

$$
\begin{gathered}
u_{t}-\Delta u=\mathscr{F} f(u), \quad(x, t) \in Q_{T}=\Omega \times(0, T] ; \\
u(x, t)=0, \quad(x, t) \in S_{T}=\partial \Omega \times[0, T] ; \\
u(x, 0)=u_{0}(x), \quad x \in \Omega,
\end{gathered}
$$

where $\mathscr{F}$ is a functional from $C(\bar{\Omega}) \rightarrow R^{1}$ defined by

$$
\int_{\mathbf{\Omega}} \mathscr{F} g(x) d x=\int_{\Gamma} g(x) d s, g(x) \in C(\bar{\Omega})
$$

where $d s$ represents the surface area element in $\Gamma$.

The problem describes chemical reaction-diffusion processes in which, due to the effects of catalyst, the reaction takes place only at some local sites. This causes the chemical concentration to be continuous, but the gradient of the concentration to have a jump at these local sites. The magnitude of the jump typically depends on the concentration. Similar phenomena are also frequently observed in biological systems, for instance on chemically active membranes. The reader is referred to [4] and [13] for

*This work is supported by NSERC Canada. 
more physical motivation. The original model equation to describe the above phenomenon was given (cf [4]) by

$$
u_{t}=\Delta u+f(u) \delta(x) \text {, in } Q_{T}=\Omega \times(0, T],
$$

where $\Omega$ contains the origin and $\delta(x)$ is the Dirac-delta (generalized) function.

The steady-state structures of solutions in one-space dimension as well as for radially symmetrical regions in higher space dimension were studied in [4] and [13]. However, the model equation (1.5) is not well-defined in a general domain of higher space dimension. To see this, we let $G(x, y ; t, \tau)$ be the Green's function with a homogeneous Dirichlet condition on the lateral boundary. Then we have the following representation for the solution of (1.6):

$$
u(x, t)=\int_{\Omega} G(x, y ; t, 0) u_{0}(y) d y+\int_{0}^{t} G(x, 0 ; t, \tau) f(u(0, \tau)) d \tau .
$$

It is known that

$$
G(x, y ; t, \tau)=Z(x, y ; t, \tau)-g(x, y ; t, \tau),
$$

where $Z(x, y ; t, \tau)$ is the fundamental solution of the heat equation and $g(x, y ; t, \tau)$ is the solution of the heat equation with the boundary value $Z(x, y ; t, \tau)$.

In a neighbourhood of $x=0, g(x, 0 ; t, \tau)$ is a smooth function but $G(x, 0 ; t, \tau)$ has the singularity $(t-\tau)^{-N / 2}$. Hence, for any $t>0$, by $(1.6), u(x, t)$ is not defined at $x=0$ if $N>1$. Consequently, the generalized function $\delta(x)$ cannot act on the function $f(u(x, t))$. It follows that $u(x, t)$ cannot satisfy the equation (1.1) in any sense unless the space dimension is equal to 1 . In order to model the described phenomenon in several space dimensions, we are motivated by the fact, for $x \in R$,

$$
\delta(x)=\frac{d}{d x} H(x),
$$

where $H(x)$ is the Heaviside function. In several space dimensions if $\Gamma=\partial A$, where $A$ is a subdomain of $\Omega$, the natural generalization of the reaction term would be to replace $\delta(x)$ by

$$
\frac{\partial}{\partial n(x)} \chi_{A}(x)
$$

where $\nabla_{n(x)}$ denotes the inward normal derivative at the boundary $x \in \partial A$ and $\chi_{A}(x)$ is the characteristic function. Then for any continuous function $g(x)$ we have (assume $A \cap \partial \Omega=$ empty):

$$
\begin{aligned}
\int_{\mathbf{\Omega}} \nabla_{n(x)} \chi_{A}(x) g(x) d x & =-\int_{\mathbf{\Omega}} \chi_{A}(x)\left(g(x) n_{i}(x)\right)_{x_{i}} d x \\
& =\int_{\partial A} g(x) d s
\end{aligned}
$$


where we have extended the normal vector $\{n(x)\}$ to a vector field defined in the whole domain $\Omega$. Thus the functional (1.4) is the natural extension for $\Gamma$ an arbitrary $C^{2}$-hypersurface in $\Omega$.

The solution of the problem (1.1)-(1.4) is defined as follows:

Definition. A continuous function $u(x, t)$ defined on $\bar{Q}_{T}$ is said to be a weak solution of $(1.1)-(1.4)$, if $u(x, t)$ satisfies

$$
\iint_{\Omega_{T}}\left[u \psi_{t}+u \Delta \psi\right] d x d t-\int_{0}^{T} \int_{\Gamma} f(u(x, t)) \psi(x, t) d s d t+\int_{\Omega} u_{0}(x) \psi(x, 0) d x=0
$$

for any test function $\psi(x, t) \in C^{2,1}\left(\bar{Q}_{T}\right)$ with $\psi(x, t)=0$ on $S_{T}$ and $\psi(x, T)=0$.

It is easy to see (cf. [10]), by choosing $\psi(x, t)$ properly, that a solution of (1.1)-(1.4) is equivalent to a solution of the following problem if $u(x, t)$ is suitably smooth:

$$
\begin{aligned}
& u_{t}-\Delta u=0, \quad \text { in } Q_{T} \backslash \Gamma ; \\
& u(x, t)=0, \quad(x, t) \in S_{T} ; \\
& u(x, 0)=u_{0}(x), \quad x \in \Omega ;
\end{aligned}
$$

and on the interface $\Gamma_{T}=\Gamma \times[0, T]$

$$
\begin{gathered}
u^{-}(x, t)=u^{+}(x, t), \\
\frac{\partial u^{-}(x, t)}{\partial n(x)}-\frac{\partial u^{+}(x, t)}{\partial n(x)}=f(u(x, t)),
\end{gathered}
$$

where $n(x)$ is the normal direction at $x \in \Gamma$ (pointing in either direction, but fixed once this direction is chosen) and

$$
\frac{\partial u^{ \pm}(x, t)}{\partial n(x)}=\lim _{z \rightarrow 0^{ \pm}} \frac{[u(x+z n(x), t)-u(x, t)]}{z} .
$$

This is a reformulation of the problem (1.1)-(1.4) in a more traditional way. In the sequel we shall study the problem in the form of (1.7)-(1.11). Once we obtain a solution of $(1.7)-(1.11)$, we then obtain a solution of $(1.1)-(1.4)$. On the other hand, in deriving energy estimates, we sometimes do use the equation (1.1) for simplicity (see Remark 1 below).

In the present paper we shall study the global solvability and finite time blowup for the problem (1.1)-(1.4). Comparing the reaction-diffusion equation

$$
u_{i}-\Delta u=u^{p}, \quad(p>1),
$$


with ours, the equation (1.1) illustrates some interesting differences. For example, it is known that in one space dimension for a regular reaction-diffusion equation, the blowup set may be a single or several points depending on the sign of the derivative of initial data. However, for the solution of $(1.1)-(1.4)$, we show that for $f(u)=u^{p}$ the blowup set consists of a single point regardless of the sign of derivative of the initial value. Moreover, we show that the blowup rate (lower bound) is

$$
u(x, t) \sim C(T-t)^{-1 /(2(p-1))}
$$

as $t$ tends to the blowup time $T$. This growth rate is the same as that for the heat equation with a nonlinear boundary condition (cf. [6] for $N=1$, also see [17] for $N>1$ ):

$$
\frac{\partial u(x, t)}{\partial n(x)}=u^{p}
$$

This is not surprising if one thinks of the equivalent problem (1.7)-(1.11). Therefore, our problem (1.1)-(1.4) is more or less similar to the heat equation with a nonlinear boundary condition. The global solvability is based on various a priori energy estimates. The blowup property in finite time is derived by using Levine's convexity method ([11]). By exploiting the maximum principle, we establish that the blowup occurs only at the interface for the case of one space dimension.

The plan of the paper is as follows. The following section deals with local and global existence. The finite time blowup is shown in Section 3. Section 4 is devoted to the study of the blowup rate and the blowup set.

\section{Local and global solvability}

Throughout this paper, $C$ denotes a generic constant whose dependency will be specified at the end of the proof. The following conditions on the data are assumed throughout the paper:

$\mathbf{H}(\mathbf{A}): u_{0}(x) \in C^{\alpha}(\bar{\Omega}) \cap C^{2+\alpha}(\Omega \backslash \Gamma)$ with $u_{0}(x)=0$ on $\partial \Omega$. The function $f(u)$ is differentiable in $[0, \infty)$. There is no intersection point in which the normal of the hypersurace $\Gamma$ and the normal of the boundary of the domain $\Omega$ lies in the same direction.

The local existence is quite standard. There are many ways to prove it. Here we sketch the proof via the Schauder fixed point theorem. Let

$$
K=\left\{v(x, t): v(x, t) \in C(\bar{\Omega}),\|v(x, t)\|_{0} \leqq K_{0}\right\},
$$

where $K_{0}$ will be determined later. If we replace the function $f(u(x, t))$ by $f(v(x, t))$ in 
(1.11) and solve $(1.7)-(1.11)$ for $u$, it is a linear diffraction problem which has a unique solution (cf. [10, Section 13, Chapter 3])

$$
u(x, t ; v) \in C^{\alpha}\left(\bar{Q}_{T}\right) \cap C^{2+\alpha, 1+(\alpha / 2)}\left(Q_{T} \backslash \Gamma_{T}\right),
$$

where $\alpha \in(0,1)$ is a constant depending on the known data. Moreover, it is differentiable up to the interface $\Gamma_{T}$ since $\Gamma$ is $C^{2}$-hypersurface. Then we can define a mapping

$$
M: v \in K \rightarrow u(x, t ; v) \in C^{\alpha}(\bar{\Omega}) .
$$

Since the embedding operator from $u(x, t ; v) \in C^{\alpha}\left(\bar{Q}_{T}\right)$ into $C\left(\bar{Q}_{T}\right)$ is compact, it follows that the mapping $M$ is compact. The continuity of $M$ can be shown using the same method as that of Theorem 2.5 (see below). To apply the Schauder fixed point theorem, we only need to show that $M$ maps $K$ into itself. This can be done, provided that $T$ is small. Indeed, by applying the maximum principle, one obtains

$$
\|u\|_{L^{\infty}\left(Q_{T}\right)} \leqq C\left(K_{0}\right),
$$

where $C\left(K_{0}\right)$ depends on $K_{0}, u_{0}$, the upper bound of $T, \Omega, \Gamma$.

We may assume that $\Gamma$ separates the domain $\Omega$ into two regions (otherwise extend it). If one modifies equation (1.7) in one of the regions as follows:

$$
u_{t}-\nabla[\nabla u+f(v(x, t)]=-\nabla f(v(x, t)),
$$

then this new equation with the heat equation in the other region becomes a diffraction problem (see Section 13 of Chapter 3 in [10]). It follows by Theorem 10.1 in Chapter 3 of $[10]$ that

$$
\|u\|_{c^{\left.\alpha_{(} \bar{Q}_{r}\right)}} \leqq C\left(K_{0}\right),
$$

where $C\left(K_{0}\right)$ and $\alpha \in(0,1)$ depend on $K_{0}$, the upper bound of $T$ as well as other known data. It follows that

$$
\|u(x, t)\|_{0} \leqq\left\|u_{0}(x)\right\|_{0}+t^{a / 2} C\left(K_{0}\right) \leqq K_{0},
$$

if we choose $K_{0}=1+\left\|u_{0}\right\|_{0}$ and restrict $T \leqq C\left(K_{0}\right)^{-2 / a}$. This implies that the mapping $M$ is from $K$ into $K$.

Finally, the Schauder fixed point theorem gives the existence of a solution of (1.7)(1.11). Uniqueness is also easy to prove (cf. Theorem 2.3 below). Thus, we have:

Theorem 2.1. Under the condition $H(A)$, the problem (1.1)-(1.4) admits a unique solution for some $T>0$.

To obtain a global solution, we need to derive an a priori bound of $u(x, t)$. This can be achieved by energy estimates. 
Theorem 2.2. Under the assumption $H(A)$, if there exists a constant $C_{0}$ such that

$$
f(u) u \leqq C_{0}\left[u^{2}+1\right]
$$

then the problem (1.1)-(1.4) admits a global solution.

Proof. In what follows, if $\Gamma$ is only a hypersurface which does not separate $\Omega$ into two regions, we can always extend $\Gamma$ in such a way that the extended curve does divide $\boldsymbol{\Omega}$ into two regions. Moreover, we require that the extended curve is also Lipschitz continuous so that we can apply the divergence theorem. On the extended part, the solution as well as its derivatives is continuous. Consequently, there is no contribution on this paprt during the performance of integration by parts. In the sequel we will always use this fact without explanation.

Let $m \geqq 2$ be even. We multiply the equation (1.7) by $u^{m+1}$ and integrate over $Q_{r}$. After performing integration by parts and using (1.10)-(1.11), we have

$$
\begin{aligned}
& \frac{1}{m+2} \int_{\Omega} u^{m+2}(x, t) d x+(m+1) \int_{Q_{t}} u^{m} u_{x}^{2} d x d t \\
& \quad=\frac{1}{m+2} \int_{\Omega} u_{0}^{m+2}(x) d x+\int_{0}^{t} \int_{\Gamma} f(u) u^{m+1} d s d t \\
& \quad \leqq \frac{1}{m+2} \int_{\Omega} u_{0}^{m+2}(x) d x+C \int_{0}^{t} \int_{\Gamma}\left[u^{m+2}+u^{m}\right] d s d t \\
& \leqq \frac{1}{m+2} \int_{\Omega} u_{0}^{m+2}(x) d x+(m+2) C+C \int_{0}^{t} \int_{\Gamma} u^{m+2} d s d t,
\end{aligned}
$$

where at the final step we have used Hölder's and Young's inequalities in the form:

$$
\begin{aligned}
\int_{0}^{t} \int_{\Gamma} u^{m} d s d t & \leqq\left(\int_{0}^{t} \int_{\Gamma} u^{m+2} d s d t\right)^{m /(m+2)}\left(\int_{0}^{t} \int_{\Gamma} d s d t\right)^{2 /(m+2)} \\
& \leqq\left(\int_{0}^{t} \int_{\Gamma} u^{m+2} d s d t+C(m+2)\right.
\end{aligned}
$$

Now we need the following trace-type interpolation inequality (cf. [12, p. 265]): for $u \in H^{1}(\Omega)$,

$$
\int_{\Gamma} u^{2} d x \leqq \varepsilon \int_{\Omega} u_{x}^{2} d x+\frac{C}{\varepsilon} \int_{\Omega} u^{2} d x
$$


Applying the above inequality for $v(x, t)=u^{m+2 /(2)}(x, t)$ to the final term in $(2.1)$, we have

$$
\begin{aligned}
& \frac{1}{m+2} \int_{\Omega} u^{m+2}(x, t) d x+(m+1) \iint_{Q_{t}} u^{m} u_{x}^{2} d x d t \\
& \quad \leqq \frac{1}{m+2} \int_{\Omega} u_{0}^{m+2}(x) d x+(m+2) C+C \varepsilon \int_{0}^{t} \int_{\Omega}\left[u^{m+2 /(2)}\right]_{x}^{2} d x d t+\frac{C}{\varepsilon} \int_{0}^{t} \int_{\Omega} u^{m+2 /(2)} d x d t
\end{aligned}
$$

It follows by taking $\varepsilon=m+1 /\left(2(m+2)^{2} C\right)$ that

$$
\begin{aligned}
& \frac{1}{m+2} \int_{\Omega} u^{m+2}(x, t) d x+\frac{(m+1)}{2} \iint_{Q_{t}} u^{m} u_{x}^{2} d x d t \\
& \leqq \frac{1}{m+2} \int_{\Omega} u_{0}^{m+2}(x) d x+(m+2) C+C(m+2) \int_{0}^{t} \int_{\Omega} u^{m+2 /(2)} d x d t,
\end{aligned}
$$

where the constant $C$ is independent of $m$. By using the well-known Alikakos iteration technique (see [6, Lemma 3.2, pp. 1730-1732], for details), we can deduce that

$$
\|u(\cdot, t)\|_{L^{\infty}(\Omega)} \leqq C .
$$

Now we can apply $W_{p}^{2,1}$-estimates (cf. [10]) to obtain

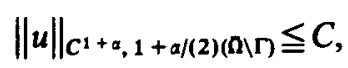

where $\alpha \in(0,1)$ is arbitrary and $C$ depends only on the known data. Finally, by the local solvability we can establish existence on $Q_{T}$ for any $T>0$. This completes our proof.

Remark 1. In deriving (2.1), one can multiply the equation (1.1) by $u^{m+1}$ and then integrate over $Q_{T}$. Formally, by assuming $u(x, t)$ is smooth in $Q_{T}$ one can apply the integration by parts and obtain the same estimates. In what follows, we shall use this way to calculate the energy.

The next two results deal with the comparison principle and the continuous dependence on the known data.

Theorem 2.3. Let $u_{0}(x)$ and $u_{0}^{*}(x)$ be two initial data which satisfy the hypothesis $H(A)$. Let $u(x, t)$ and $u^{*}(x, t)$ be the corresponding solutions in $Q_{T}$. Then, if $u_{0}(x) \geqq u_{0}^{*}(x)$ on $\bar{\Omega}$,

$$
u(x, t) \geqq u^{*}(x, t)
$$

for all $(x, t) \in \bar{Q}_{\boldsymbol{T}}$. 
Proof. This can be shown by the classical maximum principle. Indeed, if $w(x, t)=u(x, t)-u^{*}(x, t)$, then $w(x, t)$ satisfies the heat equation (1.7). Moreover, $w(x, t) \geqq 0$ on the parabolic boundary $\partial_{p} Q_{r}$. We claim that $w(x, t)$ cannot attain its negative minimum on the interface $\Gamma_{T}$. To see this we note that on the interface $\Gamma_{T}, w(x, t)$ satisfies

$$
w^{-}(x, t)=w^{+}(x, t), \frac{\partial w^{-}(x, t)}{\partial n(x)}-\frac{\partial w^{+}(x, t)}{\partial n(x)}=f^{\prime}(\theta(x, t)) w(x, t)
$$

where $\theta(x, t)$ lies between $u(x, t)$ and $u^{*}(x, t)$.

We first assume that $u_{0}(x)>u_{0}^{*}(x)$ on $\Gamma$. Then the continuity of the solution implies that there exists a $T_{0}>0$ such that $w(x, t)>0$ on $\Gamma \times\left[0, T_{0}\right]$. Let $T^{*}=$ $\sup (t: w(x, \tau)>0,0 \leqq \tau \leqq t, x \in \Gamma\}$. If $T^{*} \geqq T$, nothing needs to be proved. If $T^{*}<T$, then $w(x, t)>0$ for all $(x, t) \in \Gamma \times\left[0, T^{*}\right)$ and there exists a $x_{0} \in \Gamma$ such that $w\left(x_{0}, T^{*}\right)=0$. The maximum principle implies $w(x, t) \geqq 0$ in $\bar{Q}_{T^{*}}$. Since $w\left(x_{0} T^{*}\right)=0$, it follows that on $\bar{Q}_{T^{*}}, w(x, t)$ attains its minimum at $\left(x_{0}, T^{*}\right)$. Hopf's lemma implies that

$$
\frac{\partial w^{-}\left(x_{0}, T^{*}\right)}{\partial n\left(x_{0}\right)}<0, \frac{\partial w^{+}\left(x_{0}, T^{*}\right)}{\partial n\left(x_{0}\right)}>0
$$

This contradicts the condition (2.2) at $\left(x_{0}, T^{*}\right)$. To remove the strict inequality between the two initial values, we can use $u_{0}(x)+\varepsilon$ as the initial value which satisfies the strict inequality $u_{0}(x)+\varepsilon>u_{0}^{*}(x)$. Then we have

$$
u_{\varepsilon}(x, t) \geqq u^{*}(x, t)
$$

By taking the limit and noting the continuity of $u_{\varepsilon}$ on $\varepsilon$ (see Theorem 2.5 below), we obtain the desired result.

Corollary 2.4. Assume that $f(0) \geqq 0$. If $u_{0}(x) \geqq 0$, then $u(x, t) \geqq 0$ on $\bar{Q}_{r}$.

Theorem 2.5. Let $u_{0}(x)$ and $u_{0}^{*}(x)$ be two initial data which satisfy the condition $H(A)$. Let $u(x, t)$ and $u^{*}(x, t)$ be the corresponding solutions in $Q_{T}$. Then

$$
\left\|u-u^{*}\right\|_{0} \leqq C\left\|u_{0}-u_{0}^{*}\right\|_{0}
$$

where $C$ depends only on $\left\|u_{0}\right\|_{L^{\infty}\left(Q_{T}\right)},\left\|u_{0}^{*}\right\|_{L^{\infty}\left(Q^{T}\right)}$ as well as on $Q_{T}, \Gamma$ and $n$.

Proof. By Green's representation, we have

$$
u(x, t)=\int_{\Omega} G(x, y ; t, 0) u_{0}(y) d y+\int_{0}^{t} \int_{\Gamma} G(x, y ; t, \tau) f(u(y, \tau)) d s d \tau
$$

and similarly for $u^{*}(x, t)$. To verify the representation (2.3), we again need to extend the 
hypersurface such that $\Gamma$ separates $\Omega$ into two parts. We may assume that $A$ is one of the regions for which $n(x)$ on $\Gamma$ is the inward normal. Then the equation (1.7) as well as $(1.10)-(1.11)$ can be written in the weak sense:

$$
u_{t}-\Delta=\nabla_{n(x)} \chi_{A}(x) f(u) .
$$

Now using the Green's representation and applying the divergence theorem as before we have the expression (2.3).

Note that

$$
|G(x, y ; t, \tau)| \leqq C\left[\frac{1}{\sqrt{t-\tau}}\right]^{N} \exp \left\{-c \frac{|x-y|^{2}}{t-\tau}\right\}
$$

and

$$
\int_{\Gamma}\left[\frac{1}{\sqrt{t-\tau}}\right]^{N} \exp \left\{-c \frac{|x-y|^{2}}{t-\tau}\right\} d s \leqq \frac{C}{\sqrt{t-\tau}}
$$

It follows that

$$
\left\|u-u^{*}\right\|_{0} \leqq C\left\|u_{0}-u_{0}^{*}\right\|_{0}+C \int_{0}^{t} \frac{\left\|u-u^{*}\right\|_{0}}{\sqrt{t-\tau}} d \tau .
$$

Consequently, Gronwall's inequality yields the desired inequality.

\section{Blowup property}

To prove the blowup property, in addition to the condition $H(A)$, we need the following hypotheses:

$\mathbf{H}(\mathbf{B}):$ With $F(u)=\int_{0}^{u} f(s) d s$,

(1) $-\frac{1}{2} \int_{\Omega} u_{0 x}^{2} d x+\int_{\Gamma} F\left(u_{0}(x)\right) d s>0$;

(2) $f(u) \geqq 0$ for $u \geqq 0$. There exists a constant $a>0$ such that $f(u) u \geqq$ $2(1+a) F(u)$ for $u \geqq 0$.

The condition $H(B)(1)$ indicates that, initially, the energy is positive. The condition $H(B)(2)$ implies that the nonlinear reaction should be fast in order to have the blowup phenomenon.

Theorem 3.1. Under the assumptions $H(A)$ and $H(B)$, the solution of $(1.1)-(1.4)$ blows up in finite time.

Proof. The method follows from H. Levine's convexity method (cf. [11]). Multiply- 
ing the equation (1.1) by $u(x, t)$ and $u_{t}(x, t)$, respectively, (see Remark 1$)$ we have, after integrating over $\Omega$, that

$$
\begin{aligned}
& \frac{1}{2} \frac{d}{d t} \int_{\Omega} u^{2} d x+\int_{\Omega} u_{x}^{2} d x=\int_{\Gamma} f(u) u d s \\
& \int_{\Omega} u_{t}^{2} d x+\frac{1}{2} \frac{d}{d t} \int_{\Omega} u_{x}^{2} d x=\int_{\Gamma} f(u) u_{t} d s
\end{aligned}
$$

We define

$$
J(t)=-\frac{1}{2} \int_{\Omega} u_{x}^{2} d x+\int_{\Gamma} F(u(x, t)) d s
$$

From (3.2), we have

$$
J(t)-J(0)=\int_{0}^{t} \int_{\Omega} u_{t}^{2} d x d t
$$

Let

$$
I(t)=\int_{0}^{t} \int_{\Omega} u^{2} d x d t+A,
$$

where $A$ is a constant to be specified later.

Then

$$
I^{\prime}(t)=\int_{\Omega} u(x, t)^{2} d x, \quad I^{\prime \prime}(t)=\frac{d}{d t} \int_{\Omega} u^{2} d x
$$

From (3.1) and the assumption $H(B)$, one obtains

$$
I^{\prime \prime}(t) \geqq 4(1+a) J(t) .
$$

For $\alpha>0$ sufficiently small, to be chosen later, we calculate

$$
\begin{aligned}
& I^{\prime \prime}(t) I(t)-(1+\alpha) I^{\prime}(t)^{2} \\
& \geqq 4(1+a) J(t) I(t)-(1+\alpha) I^{\prime}(t)^{2} \\
& \quad=4(1+a)\left[J(0)+\int_{0}^{t} \int_{\Omega} u_{t}^{2} d x d t\right]\left[\int_{0}^{t} \int_{\Omega} u^{2} d x d t+A\right]-(1+\alpha) I^{\prime}(t)^{2}
\end{aligned}
$$

Now 


$$
\begin{aligned}
I^{\prime}(t) & =\int_{\mathbf{\Omega}} u^{2} d x \\
& =2 \int_{0}^{t} \int_{\mathbf{\Omega}} u u_{t} d x d t+\int_{\mathbf{\Omega}} u_{0}^{2} d x \\
& \leqq 2\left[\int_{0}^{t} \int_{\mathbf{\Omega}} u^{2} d x d t\right]^{1 / 2}\left[\int_{0}^{t} \int_{\mathbf{\Omega}}^{2} u_{t}^{2} d x d t\right]^{1 / 2}+\int_{\mathbf{\Omega}} u_{0}^{2} d x
\end{aligned}
$$

by Hölder's inequality.

It follows by Cauchy's inequality that

$$
I^{\prime}(t)^{2} \leqq 4(1+\varepsilon) \int_{0}^{t} \int_{\Omega} u^{2} d x d t \int_{0}^{t} \int_{\Omega} u_{t}^{2} d x d t+\left(1+\frac{1}{\varepsilon}\right)\left[\int_{\Omega} u_{0}^{2}(x) d x\right]^{2}
$$

Hence,

$$
\begin{aligned}
& I^{\prime \prime}(t) I(t)-(1+\alpha) I^{\prime}(t)^{2} \\
& \quad \leqq 4(1+\alpha)\left\{1-\frac{(1+\alpha)(1+\varepsilon)}{1+a}\right\}\left[\int_{0}^{t} \int_{\Omega} u_{t}^{2} d x d t\right]\left[\int_{0}^{t} \int_{\Omega} u^{2} d x d t\right] \\
& +4(1+\alpha) A \int_{0}^{t} \int_{\Omega} u_{t}^{2} d x d t+4(1+a) J(0)\left[\int_{0}^{t} \int_{\Omega} u^{2} d x d t+A\right] \\
& -(1+\alpha)\left(1+\frac{1}{\varepsilon}\right)\left[\int_{\Omega} u_{0}^{2} d x\right]^{2}
\end{aligned}
$$

Consequently, if we choose $\alpha$ and $\varepsilon$ sufficiently small such that

$$
(1+\alpha)(1+\varepsilon)<1+a
$$

while we take $A$ to be large enough such that $(J(0)>0$ by $H(B))$,

$$
A \geqq \frac{(1+\alpha)\left(1+\frac{1}{\varepsilon}\right)\left[\int_{\Omega} u_{0}^{2} d x\right]^{2}}{4(1+a) J(0)},
$$

then we obtain

$$
I^{\prime \prime}(t) I(t)-(1+\alpha) I^{\prime}(t)^{2} \geqq 0 .
$$

This implies that $I(t)^{-a}$ must become zero in finite time. That is $u(x, t)$ will become infinite in finite time. Moreover, the blowup time, say $T$, obeys the following estimate (cf [11]) 


$$
T \leqq \frac{(1+\alpha)\left(1+\frac{1}{\varepsilon}\right) \int_{\Omega} u_{0}^{2} d x}{4(1+a) \alpha J(0)}
$$

Corollary 3.2. For $f(u)=u^{p}$ with $p>1$, the solution will blowup in finite time, provided that the initial data satisfies $H(B)(1)$.

Proof. It is easy to verify that the assumption $H(B)(2)$ holds for $f(u)=u^{p}$. The result follows from Theorem 3.1.

In order to obtain a result for an exponential nonlinearity, we need the condition:

H(B): (3) Let dist $\{\Gamma, \partial \Omega\}>0$. Assume that $\Delta u_{0} \geqq 0$ on $\Omega \backslash \Gamma$ and $u_{0}(x) \geqq$ $2(1+a)$ in a neighbourhood of $\Gamma$.

Corollary 3.3. For $f(u)=e^{u}$, in addition to the assumption $H(B)(1)$, if $u_{0}(x)$ satisfies $H(B)(3)$, then $u(x, t)$ blows up in finite time.

Proof. By the condition $H(B)(3)$ and the maximum principle, we can obtain $u_{t} \geqq 0$. Therefore, there exists a neighbourhood, say $K\left(\Gamma_{T}\right)$, of $\Gamma_{T}$ such that $u(x, t) \geqq 2(1+a)$. It follows that for $(x, t) \in K\left(\Gamma_{T}\right)$

$$
u e^{u} \geqq 2(1+a)\left(e^{u}-1\right)
$$

Thus, the assumption $H(B)(2)$ holds in $K\left(\Gamma_{T}\right)$, which is sufficient from the proof of Theorem 3.1. Hence, Theorem 3.1 implies the desired result.

\section{Blowup rate estimates and blowup set}

Throughout this section, $T$ will denote the blowup time.

Theorem 4.1. For $f(u)=u^{p}$ with $p>1$, there exists a constant $C>0$ such that

$$
\sup u(x, t) \geqq C(T-t)^{-(1 / 2(p-1))}
$$

$\Omega$

Proof. For any $z \in(0, T)$, we have

$$
u(x, t)=\int_{\mathbf{\Omega}} G(x, y ; t, z) u(y, z) d y+\int_{z}^{t} \int_{\Gamma} G u^{p} d s d \tau
$$

For $t \in(z, T)$, let 


$$
A(t)=\sup _{z \leqq r \leqq s}\|u(\cdot, \tau)\|_{L^{\infty}(\Omega)} .
$$

Then

$$
\begin{aligned}
A(t) & \leqq A(z)+C \int_{z}^{1} \frac{1}{\sqrt{t-\tau}} d \tau A(t)^{p} \\
& =A(z)+2 C \sqrt{t-z} A(t)^{p} .
\end{aligned}
$$

By using the argument of [15], one concludes that for all $t \in(z, T)$

$$
(t-z)^{1 / 2} \geqq \frac{\beta-A(z)}{C \beta^{p}},
$$

provided that $\beta>A(z)$ and $0<z<T$. Otherwise, from (4.1) we would have $A(t) \leqq \beta$ for all $t \in(z, T)$, which is contradiction since $u(x, t)$ blows up at $t=T$. In particular, if we take $\beta=2 A(z)$ in (4.2) and let $t \rightarrow T$ to obtain

$$
A(z) \geqq C(T-z)^{-1 /(2(p-1))} .
$$

Remark 2. It would be interesting to have a similar estimate for the upper bound. It is an open problem. However, a formal asymptotic analysis suggests the upper bound should be the same as the lower bound.

We now study the blowup set. We are only able to deal with the problem in one space dimension. In this case the model equation (1.1) reduces to (1.5) if one assumes that the interface $\Gamma=\{x=0\}$. In the following, we take the domain $Q_{T}=(-1,1) \times(0, T)$.

Recall that a point $x_{0}$ is said to be a blowup point if there exists a sequence $\left(x_{n}, t_{n}\right)$ with $t_{n}<T$ such that $\left(x_{n}, t_{n}\right) \rightarrow\left(x_{0}, T\right)$ as $n \rightarrow \infty$ and

$$
\lim _{n \rightarrow \infty} u\left(x_{n}, t_{n}\right)=\infty .
$$

Theorem 4.2. Assume $G(z)=\int_{z}^{\infty} d s / f(s)$ is defined on $\left[z_{0}, \infty\right)$ with some $z_{0}>0$ and $f^{\prime}(s) \geqq 0, f^{\prime \prime}(s)>0$ for $s>0$. Moreover, for $s \geqq s_{0}>0$ assume there exists a constant $K\left(s_{0}\right)>0$ such that

$$
\left|\frac{f^{\prime}(s)}{f(s) f^{\prime \prime}(s)}\right| \leqq K\left(s_{0}\right)
$$

then the blowup occurs only at $x=0$.

Proof. First of all, it is clear that $x=0$ is a blowup point.

Let $Q_{T}^{*}=[0,1] \times[0, T)$. On $Q_{T}^{*}$, we define a new function 


$$
w(x, t)=u(x, t)+u(-x, t)
$$

It is easy to see that $w(x, t)$ satisfies

$$
\begin{gathered}
w_{t}-w_{x x}=0, \quad(x, t) \in Q_{T}^{*} ; \\
w_{x}(0, t)=-f\left(\frac{w(0, t)}{2}\right), \quad 0 \leqq t<T ; \\
w(1, t)=0, \quad 0 \leqq t<T ; \\
w(x, 0)=w_{0}(x) \equiv u_{0}(x)+u_{0}(-x), \quad 0 \leqq x \leqq 1 .
\end{gathered}
$$

For a fixed $\alpha>0$, we can choose $t_{0}$ close to $T$ such that

$$
w(0, t) \geqq \alpha, \quad t_{0} \leqq t<T .
$$

The monotonicity of $f(s)$ implies that $w_{x}(0, t) \leqq-f(\alpha / 2)$ in $t_{0} \leqq t<T$. Consequently, there exists a neighbourhood $N_{a}(x, t)=\left\{0 \leqq x \leqq a, t_{0} \leqq t<T\right\}$ such that

$$
w_{x}(x, t) \leqq-\frac{1}{2} f\left(\frac{\alpha}{2}\right)
$$

for all $(x, t) \in N_{a}(x, t)$.

On $N_{a}(x, t)$, we introduce an auxiliary function

$$
J(x, t)=w_{x}(x, t)+v(x, t) f(w)
$$

where $v(x, t)$ will be specified later. We would like to show that there exists a bounded function $v(x, t)$ such that $J(x, t) \leqq 0$. To this end we first choose $v(x, t)$ satisfying

$$
v(0, t)=v(a, t)=0, \quad t_{0} \leqq t<T
$$

Moreover, we choose $v_{0}(x)=v\left(x, t_{0}\right)$ to be sufficiently small such that

$$
\left\|v_{0}(x)\right\|_{0} \leqq \frac{f(\alpha / 2)}{2\left\|f\left(w\left(x, t_{0}\right)\right)\right\|_{0}}
$$

With the above $v(x, t)$, we see that on the parabolic boundary $\partial_{p} N_{a}(x, t)$

$$
J(x, t) \leqq 0 .
$$


By a direct calculation, one finds

$$
J_{1}-J_{x x}=\left[v_{1}-v_{x x}\right] f(w)-2 v_{x} f^{\prime}(w) w_{x}-v f^{\prime \prime}(w) w_{x}^{2} .
$$

Since $f^{\prime \prime}(w)>0$, the Cauchy inequality implies

$$
\left|2 v_{x} f^{\prime}(w) w_{x}\right| \leqq v f^{\prime \prime}(w) w_{x}^{2}+\frac{f^{\prime}(w)}{f^{\prime \prime}(w)} \frac{v_{x}^{2}}{v}
$$

It follows from the assumptions that

$$
\left|2 v_{x} f^{\prime}(w) w_{x}\right| \leqq v f^{\prime \prime}(w) w_{x}^{2}+K\left(s_{0}\right) \frac{v_{x}^{2}}{v} f(w)
$$

Consequently,

$$
J_{t}-J_{x x} \geqq\left[v_{t}-v_{x x}-\frac{K_{0} v_{x}^{2}}{v}\right] f(w)
$$

where $K_{0}=K\left(s_{0}\right)$. Now we consider the following problem

$$
\begin{gathered}
v_{t}-v_{x x}-\frac{K_{0} v_{x}^{2}}{v}=0,(x, t) \in N_{a}(x, t), \\
v(0, t)=v(a, t)=0, \quad t_{0} \leqq t \leqq T, \\
v\left(x, t_{0}\right)=v_{0}(x), \quad 0 \leqq x \leqq a .
\end{gathered}
$$

Note that the equation (4.8) is singular at the boundary. However we claim that the problem (4.8)-(4.10) admits a bounded solution on $\left[t_{0}, T\right]$. For this purpose we consider the following regularized problem:

$$
\begin{gathered}
v_{t}-v_{x x}-\frac{K_{0} v_{x}^{2}}{v}=0,(x, t) \in N_{a}(x, t), \\
v(0, t)=v(a, t)=\varepsilon, \quad t_{0} \leqq t \leqq T, \\
v\left(x, t_{0}\right)=v_{0}(x)+\varepsilon, \quad 0 \leqq x \leqq a .
\end{gathered}
$$

The maximum principle implies

$$
\varepsilon \leqq v(x, t) \leqq M=\left\|v_{0}\right\|_{0} .
$$

Since the consistency conditions hold at the corner points $(0,0)$ and $(a, 0)$, the classical 
theory implies that the problem (4.11)-(4.13) posseses a unique classical solution $v(x, t) \in C\left(\bar{N}_{a}(x, t)\right) \cap C^{2+\beta, 1+(\beta / 2)}\left(N_{a}\right)$. Moreover, again by the maximum principle, $\left\{v_{\varepsilon}(x, t)\right\}$ is a monotone decreasing sequence. It follows that $\left\{v_{\varepsilon}(x, t)\right\}$ converges to a function, denoted by $v(x, t)$, uniformly on $N_{a}(x, t)$. Furthermore, the Schauder theory yields

$$
\left\|v_{\varepsilon}\right\|_{C^{2+\beta, 1+(\beta / 2)\left(N^{\prime}\right)}} \leqq C,
$$

where $N^{\prime}$ is any compact subdomain of $N_{a}(x, t)$ and $C$ depends only on $M, a$ and $K_{0}$, but not on $\varepsilon$.

The standard compactness argument implies that the limit function $v(x, t)$ solves the problem $(4.8)-(4.10)$. Finally, we choose $v(x, t)$ to be the solution of $(4.8)-(4.10)$, then on $N_{a}(x, t)$

$$
J_{t}-J_{x x} \geqq 0 .
$$

Combining the initial and boundary conditions, we obtain by the maximum principle that

$$
J(x, t) \leqq 0,(x, t) \in N_{a}(x, t) .
$$

Now we integrate the above inequality over $[0, a]$ to obtain

$$
\int_{0}^{a} \frac{w_{x}}{f(w)} d x \leqq-\int_{0}^{a} v(x, t) d x=-C(t) .
$$

It follows that

$$
\int_{w(0, t)}^{w(a, t)} \frac{1}{f(s)} d s \leqq-C(t)
$$

That is

$$
G(w(a, t))-G(w(0, t)) \geqq C(t) .
$$

Since $x=0$ is a blowup point, we have $G(w(0, t)) \rightarrow 0$ as $t \rightarrow T$. As $v(x, t) \geqq 0$ and $v(x, t) \neq 0$, we have for any $t \in\left[t_{0}, T\right]$

$$
C(t)=\int_{0}^{a} v(x, t) d x>0
$$

It follows that

$$
\lim _{t \rightarrow T} \inf _{t o \leqq} G(w(a, \tau)) \geqq C(T)>0
$$


which implies

$$
\lim _{t \rightarrow T} \sup _{t o \leqq \tau \leqq t} w(a, \tau)<\infty
$$

For any $x_{0} \in(0, a)$, we can repeat the above procedure to conclude that $x_{0}$ is not a blowup point. On the interval $[a, 1]$, we can use the maximum principle to get

$$
\lim _{t \rightarrow T} \sup _{t \rightarrow \leqq} w(x, t)<\infty
$$

This completes our proof.

Corollary 4.3. For $f(u)=u^{p}$ with $p>1$ or $f(u)=e^{u}$, the blowup set consists of the single point $x=0$.

Acknowledgement. The authors would like to express their gratitude to the referee for many valuable comments.

\section{REFERENCES}

1. N. D. Alikakos, $L_{p}$-bounds of solutions of reaction-diffusion equations, Comm. Partial Differential Equations 4 (1979), 827-868.

2. D. G. Aronson, Non-negative solutions of linear parabolic equations, Ann. Scuola. Norm. Sup. Pisa 22 (1968), 607-694.

3. J. Bebernes, Mathematical Problems from Combustion Theory (Applied Mathematical Sciences 83, Springer-Verlag, New York, 1989).

4. K. Bimpong-Bota, P. Ortoleva and J. Ross, Far-from-equilibrium phenomena at local sites of reaction, J. Chem. Phys. 60 (1974), 3124.

5. J. M. Chadam, A. Perice and H. M. Yin, The blowup property of solutions to a chemical diffusion equation with localized reactions, J. Math. Anal. Appl. 169 (1992), 313-328.

6. M. Fila and P. Quitrer, The blowup rate for the heat equation with a nonlinear boundary condition, Math. Methods Appl. Sci. 14 (1991), 197-205.

7. A. Friedman and B. McLeod, Blowup of positive solutions of semilinear heat equations, Indiana Univ. Math. J. 34 (1985), 425-477.

8. A. Friedman, Partial Differential Equations of Parabolic Type (Prentice-Hall, 1964).

9. Y. Giga and R. V. KонN, Asymptotic self-similar blowup of semilinear heat equations, Indiana Univ. Math. J. 34 (1987), 425-447.

10. O. A. Ladyzenskaja, V. A. Solonnikov and N. N. Ural'ceva, Linear and Quasi-linear Equations of Parabolic Type (Amos Math. Soc. Transl. 23, Providence, R.I., 1968).

11. H. A. Levine and L. E. PAYNe, Nonexistence theorems for the heat equation with nonlinear boundary conditions and for the porous medium equation backward in time, J. Differential Equations 16 (1974), 319-334.

12. V. G. Maz'JA, Sobolev Spaces (Springer-Verlag, Berlin Heidelberg, 1985). 
13. P. ORtoleva and J. Ross, Local structures in chemical reactions with heterogeneous catalysis, J. Chem. Phys. 56 (1972), 4397-4452.

14. W. WALTER, On existence and nonexistence in the large of solutions of parabolic differential equations with a nonlinear boundary condition, SIAM J. Math. Anal. 6 (1974), 85-90.

15. F. B. WeIssLer, Existence and non-existence of global solutions for a semilinear heat equation, Israel J. Math. 38 (1981), 29-40.

16. H. M. YIN, On parabolic Volterra equations in several space dimensions, SIAM J. Math. Anal. 22 (1991), 1723-1737.

17. H. M. Yin, Blowup versus global solvability for a class of nonlinear parabolic equations (University of Toronto, 1992), preprint.

Department of Mathematics

MCMASTER UNIVERSITY

HAMiLton

Ontario L8S 4K1

Canada
Department of Mathematics

UNIVERSITY OF TORONTO

TORONTO

Ontario M5S 1A1

Canada

Current Address:

Department of Mathematics UNIVERSITY OF Notre DAME

Notre DAME, IN 46556

USA 